\title{
Posterior reversible encephalopathy syndrome related to anemia correction in a patient with uterine myoma: a case report
}

\author{
Jisun Lee, Hyun Jung Lee \\ Department of Obstetrics and Gynecology, School of Medicine, Kyungpook National University, Kyungpook National University Hospital, \\ Daegu, Korea
}

\begin{abstract}
Although posterior reversible encephalopathy syndrome (PRES) is induced by various causes, a few cases have occurred after severe anemia correction. In this case report, a 45-year-old female patient visited emergency department with a chief complaint of dizziness due to severe anemia related to hypermenorrhea caused by uterine myoma. Before her operation, she had an abrupt headache and seizure during anemia correction with transfusion and injection of gonadotropin-releasing hormone agonist. Immediately after the operation, she experienced visual disturbances, followed by limb weakness and tonic-clonic movements. Magnetic resonance imaging showed alterations in parietal and occipital lobes suggesting cerebrovascular edema with hypoperfusion. Here, we presented and discussed the clinical and radiologic features of PRES related to anemia correction.
\end{abstract}

Keywords: Anemia; Blood transfusion; Magnetic resonance imaging; Posterior reversible encephalopathy syndrome

\section{Introduction}

Uterine myomas are the most common benign pelvic tumors in women. The symptoms associated with uterine myomas are heavy or prolonged menstrual bleeding, abnormal uterine bleeding, and resultant iron-deficiency anemia (IDA), pelvic pain, infertility, and/or recurrent spontaneous abortion [1]. Severe bleeding can lead to IDA of up to $2.0 \mathrm{~g} / \mathrm{dL}$ of hemoglobin $(\mathrm{Hb})$. Transfusion-related immunologic or nonimmunologic complications are widely recognized, and neurological complications have also been observed in few cases of patients who underwent anemia correction.

Posterior reversible encephalopathy syndrome (PRES) can be caused by various causes and in a small number of cases, it occurs after correction of severe anemia. However, the characteristic fea- tures of PRES after blood transfusion are unclear. In this case report, a 45-year-old female patient visited emergency department with dizziness due to severe anemia related to hypermenorrhea caused by uterine myoma. She experienced sudden headaches and seizures during anemia correction through blood transfusions, treatment with gonadotropin-releasing hormone $(\mathrm{GnRH})$ agonist, and surgical treatment to eliminate the cause of bleeding. She was diagnosed with PRES based on her clinical symptoms, such as headache, visual impairment, seizures, and distinctive magnetic resonance imaging (MRI) findings of reversible white matter edema. In this case report, we reviewed a rare case of PRES related to the correction of anemia and discussed the clinical and radiological features through a literature review.

Received: July 28, 2021 • Revised: August 25, 2021 • Accepted: August 25, 2021

Corresponding author: Hyun Jung Lee, MD, PhD

Department of Obstetrics and Gynecology, School of Medicine, Kyungpook National University, Kyungpook National University Hospital, 130 Dongdeok-ro, Jung-gu, Daegu 41944, Korea

Tel: +82-53-200-5691 • Fax: +82-53-423-7905• E-mail: hyunjunglee@knu.ac.kr 


\section{Case}

Ethical statements: The Institutional Review Board (IRB) of Kyungpook National University Hospital (IRB No: KNUH 2017-06-012) approved the retrospective data collection and analysis. Written informed consent was obtained for publication of this case report and accompanying images.

A 45-year-old female patient visited emergency department with a chief complaint of dizziness (day 1 ). The initial laboratory studies showed a hematocrit of $8.5 \%, \mathrm{Hb}$ level of $2.0 \mathrm{~g} / \mathrm{dL}$, white blood cells count of $8,500 / \mathrm{mm}^{3}$, and platelet count of $165,000 / \mathrm{mm}^{3}$ (Fig. 1). Initial systolic blood pressure (SBP) was between 110 $\mathrm{mmHg}$ and $100 \mathrm{mmHg}$, and diastolic blood pressure (DBP) was between $75 \mathrm{mmHg}$ and $60 \mathrm{mmHg}$. After the transfusion of three packs of 400-mL red blood cells (RBCs), her hematocrit and $\mathrm{Hb}$ levels were improved to $16.7 \%$ and $5.2 \mathrm{~g} / \mathrm{dL}$, respectively. Bone marrow biopsy was done to exclude hematologic diseases, and it showed hypercellular marrow particles with erythroid hyperplasia associated with IDA. Abdominal computed tomography (CT) and MRI showed a 12-cm uterine mass. Therefore, her diagnosis was severe IDA related to hypermenorrhea caused by uterine myo- ma. She received additional four more packs of 400-mL RBCs. The final laboratory study showed a hematocrit of $26.0 \%$ and an $\mathrm{Hb}$ level of $7.9 \mathrm{~g} / \mathrm{dL}$ (day 2). She was discharged with a prescription for oral iron and vitamin $\mathrm{C}$ supplement. On an outpatient follow-up, her laboratory study showed a hematocrit of $38.8 \%$ and an $\mathrm{Hb}$ level of $11.6 \mathrm{~g} / \mathrm{dL}$ (day 11, Fig. 1). She planned to undergo elective myomectomy.

One month later (day 34), she returned to the emergency department with a chief complaint of abrupt headache and seizure. The follow-up laboratory study showed a hematocrit of $38.5 \%$ and $\mathrm{Hb}$ level of $11.8 \mathrm{~g} / \mathrm{dL}$. Although the noncontrast CT scan showed no definite lesions in the brain, the findings of fluid-attenuated inversion recovery (FLAIR) MRI showed a focal cortical swelling with signal changes in the left occipital and parietal lobes, which were normalized 1 week after based on a follow-up MRI (Fig. 2). After 1 week in a neurology department, her operation schedule was delayed for 2 months to avoid the acute phase of newly diagnosed neurological problems.

While waiting for 2 months, she was injected with two doses of $3.75 \mathrm{mg}$ GnRH agonist every month to prevent IDA caused by excessive bleeding during menstruation (day 36). On preoperative laboratory studies (day 60), the hematocrit and $\mathrm{Hb}$ level were $42.9 \%$ and $14.1 \mathrm{~g} / \mathrm{dL}$, respectively. No neurological events were ob-

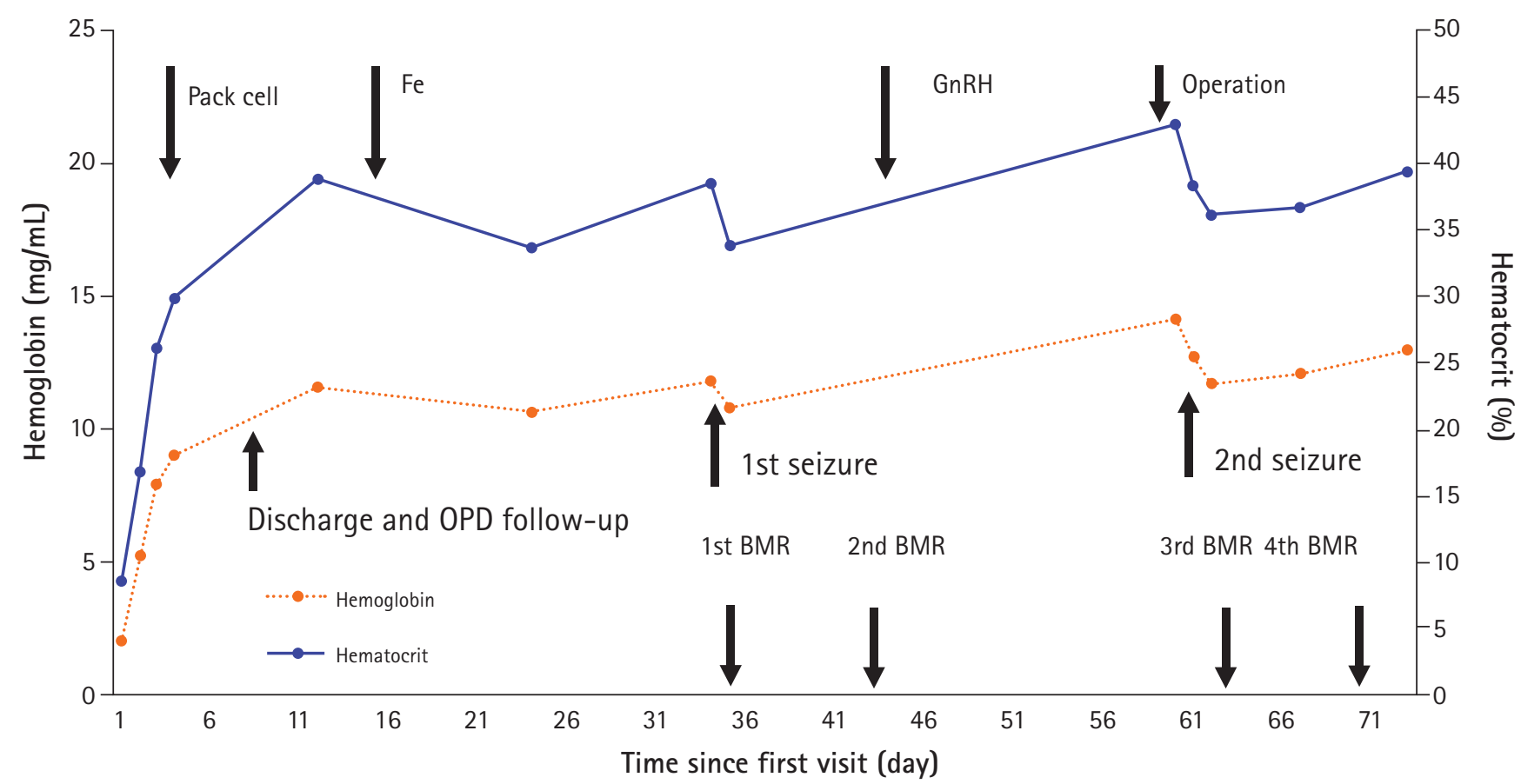

Fig. 1. A 45-year-old female patient with anemia. Her hemoglobin and hematocrit levels were followed up after her first visit to an emergency department with a chief complaint of dizziness. The clinical flow chart shows that transfusions were performed within 3 days. The first seizure occurred on day 34, while the second seizure occurred on day $61 . \mathrm{GnRH}$, gonadotropin-releasing hormone; OPD, outpatient department; BMR, brain magnetic resonance imaging. 

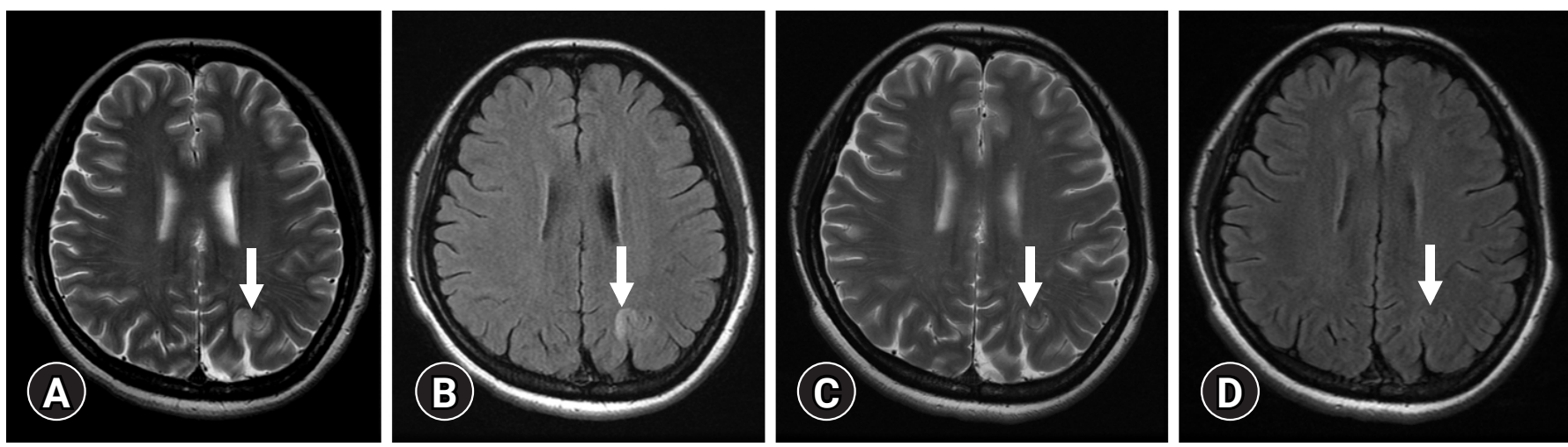

Fig. 2. The magnetic resonance imaging findings after the first seizure. (A) The T2-weighted image (T2WI) and (B) fluid-attenuated inversion recovery (FLAIR) show an increased signal intensity (arrows) in the gray and white matter of the left parietal lobes. The followup (C) T2WI and (D) FLAIR after 1 week reveal the disappearance (arrows) of the previous lesions without any complications.

served during the 2 months. During the operation, her blood pressure remained within the normal range, and the other vital signs were stable. Initial SBP was between $130 \mathrm{mmHg}$ and $80 \mathrm{mmHg}$, and DBP was between $80 \mathrm{mmHg}$ and $40 \mathrm{mmHg}$ (day 61).

One day after the surgery (day 62), she experienced visual disturbances, followed by limb weakness and tonic-clonic movements. On the second day after surgery, her neurologic symptoms, such as headache, vision change, paresis, hemianopsia, nausea, and altered mental status, were aggravated. The patient's mean DBP was measured as high as $99 \mathrm{mmHg}$, and after treatment, DBP dropped to an average of $73 \mathrm{mmHg}$. After 2 days of controlling the blood pressure, the patient's neurologic symptoms were improved. Her Glasgow Coma Scale score was 12. The patient's clinical flow chart (Fig. 1) shows that transfusions were performed within three days and anemia was corrected abruptly. The first seizure occurred on day 34 , while the second seizure occurred on day 61 . The $\mathrm{Hb}$ and hematocrit levels rapidly increased immediately after the blood transfusions, gradually increased with GnRH administration, and then decreased after the seizures.

After checking the subtle hypodensity in the occipital lobe on a noncontrast CT scan, MRI was immediately performed. The T2-weighted image (T2WI) showed a high signal intensity on the subcortical white matter of the bilateral temporo-occipital lobes, which showed isosignal intensities on diffusion-weighted images (DWI) and high signal intensities on the apparent diffusion coefficient (ADC) maps. The mean transit time in the affected lesion had an increased value on perfusion MRI (Fig. 3). After 1 week, her symptoms, such as headache, visual field damage, and vomiting, were improved, and the lesion was diminished on a follow-up MRI (supporting data, Supplementary Fig. 1). One month later, an outpatient follow-up examination showed that most neurological defects had recovered, except for some visual field defects (day 73).

\section{Discussion}

PRES was first described in 1996 by Hinchey et al. [2]. It is a clinical syndrome characterized by symptoms, such as headache, seizures, altered consciousness, and visual disturbances [3]. It commonly involves the white matter in the bilateral parieto-occipital lobes, brain stem, cerebellum, basal ganglia, and frontal lobes $[4,5]$. Although PRES is induced by various causes, including severe hypertension, eclampsia/preeclampsia, immunosuppressive medications, and various causes of renal failure, a small number of cases have also occurred after the correction of severe anemia. According to a recent literature review, there are 21 studies about PRES related to blood transfusion, of which five are related to uterine fibroids [6].

Although the pathophysiology of transfusion-associated PRES has not been clearly defined, several theories have been proposed. The mechanism can be inferred from the rapid increase in blood viscosity and hematocrit and $\mathrm{Hb}$ levels, which may exacerbate endothelial cell damages, blood-brain barrier dysfunction, and vasogenic edema, resulting in PRES despite a normal systemic blood pressure. Blood transfusion may cause a rapid increase in total blood volume, which further leads to cerebral blood flow overload. However, the first onset of neurological symptoms after blood transfusion was after 31 days. Blood transfusions were performed during the initial 3 days in the emergency department. Then, only iron preparations were used to correct the anemia. According to a recent literature review, the average onset of neurological symptoms after a blood transfusion was after 7.1 days, ranging from 1 to 18 days [7].

However, PRES is commonly associated with acute hypertension related to hypervolemia [2]. Cerebral hyperperfusion exceeding the autoregulatory capacity of cerebral capillary perfusion pressure might result in vasogenic edema found in PRES [8]. Severe 

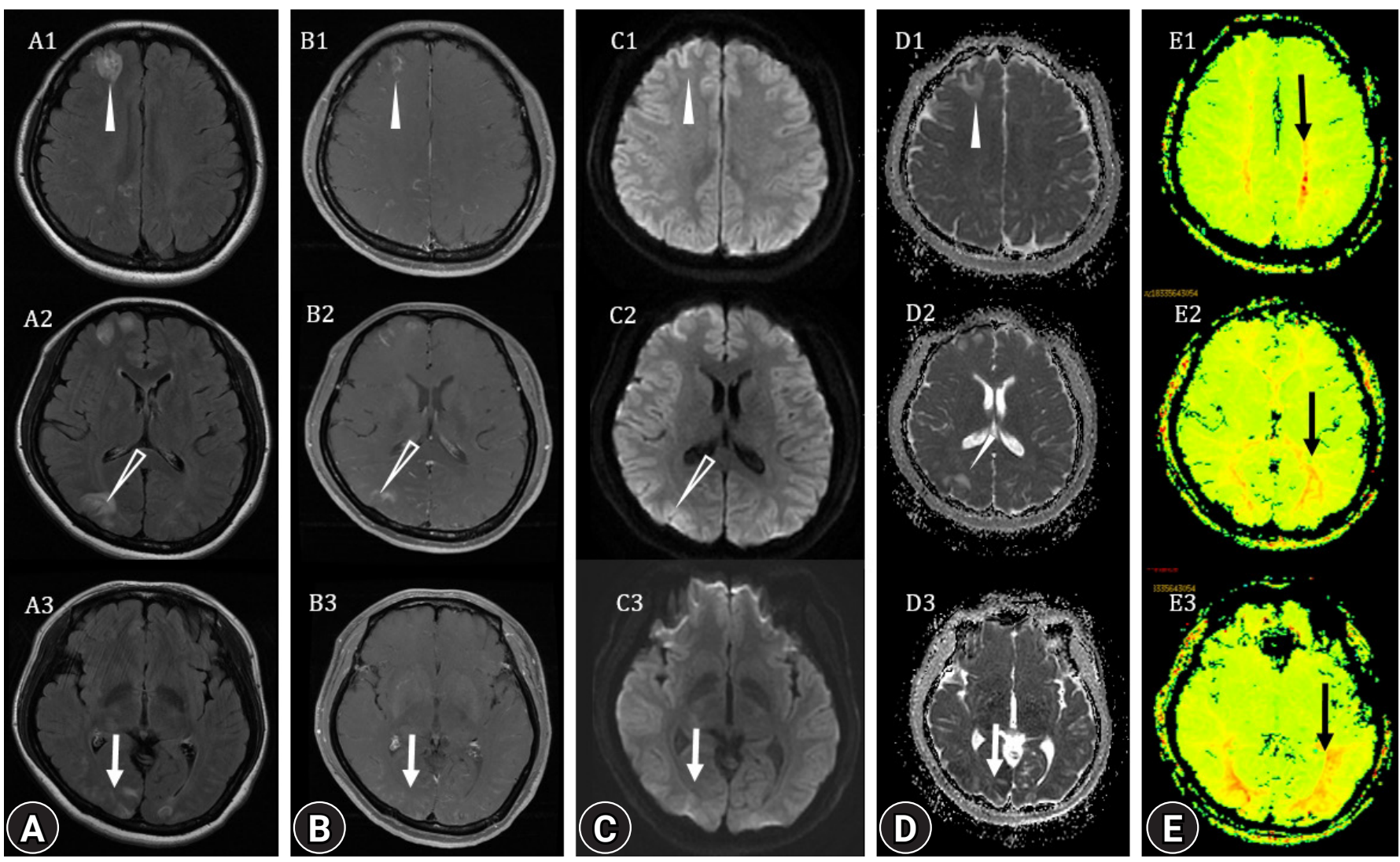

Fig. 3. After 2 months, the magnetic resonance imaging (MRI) findings show the extensive signal change in the left frontal (white arrowheads), parietal (empty arrowheads) and occipital (white arrows) lobes and right occipital lobe at the level of centrum semiovale, foremen Monro and occipital horn of lateral ventricle (1, upper; 2, middle; 3, lower raw) on (A) T2-weighted image, (B) contrastenhanced T1-weighted image, (C) diffusion-weighted image, and (D) apparent diffusion coefficient map. (E) MRI perfusion shows an increase in mean transit time mainly in the white matter of the parietal and occipital lobes (black arrows), suggesting hypoperfusion.

anemia can also be a predisposing factor because an inadequate oxygen supply to the brain may result in endothelial cell dysfunction, further causing a functional loss or damage to the integrity of the blood-brain barrier in capillary circulation.

The patient was scheduled for myomectomy to eliminate the cause of bleeding, and GnRH agonist was administered to control the temporary bleeding. During the operation, the uterus was removed, along with the fibroids, without any procedural problems. A seizure occurred immediately after the operation, and an expanded range of PRES was confirmed in an MRI acquired immediately. Recurrent episodes of PRES are rare, and its incidence is not known. To the best of our knowledge, there was only one case report about recurrent PRES related to anemia correction.

Recently, GnRH agonist therapy has been added as a factor associated with PRES [9]. Estrogen is known to exert protective effects on the vascular endothelium against ischemia-reperfusion injury in various organs [10]. GnRH agonists induce hypoestrogenic status which is attenuating the protective effects of estrogen on vascular endothelium, and consequently may play a role in the develop- ment of PRES. However, considering that the incidence of PRES is very low despite the high frequency of prescribing GnRH agonists, it is highly likely that the drug had a secondary effect on the increasing $\mathrm{Hb}$ concentration by preventing bleeding, and not as a direct cause of PRES occurrence.

The MRI findings of this patient showed high signal intensities on T2WIs and ADC maps, which were compatible to the signs of vasogenic edema. Although most cases of PRES are transient and reversible, there are also irreversible cases such as a form of hemorrhage or infarction. DWI can be helpful in differentiating between arterial ischemic injury (reduced ADC) and vasogenic brain edema (increased $\mathrm{ADC}$ ) that is characteristic in patients with PRES [11].

The presented patient's perfusion images showed extensive high perfusion in the bilateral posterior circulatory region, supporting the proposed hypothesis. Hypertension/hyperperfusion theory is favored in explaining the mechanism of PRES due to the common presence of blood pressure elevation and response to high blood pressure management. However, the key issue of this theory remains controversial because PRES can also occur in patients with 
normal blood pressure, failure of toxicity blood pressure reaching the limit of autoregulation, and brain edema can be lower in patients with severe hypertension $[12,13]$. In our case, the patient was hospitalized with generalized seizure and hypertension. Our patient's MRI showed a sulcal enhancement, and the FLAIR image also showed hyperintense acute reperfusion marker findings, which were not bleeding because there was no signal attenuation in the $\mathrm{T} 2$ gradient echo image [14].

In summary, we present a case of PRES related to anemia correction in a patient with severe anemia due to uterine bleeding caused by myoma. The mechanism could be related to autoregulatory failure, cytotoxic effects of acute anemia correction on vascular endothelia, and altered cerebral blood flow. GnRH agonist treatment should also be considered as another trigger of PRES. Although these cases are extremely rare, it should be considered as one of the side effects during treatment of patients with severe anemia patients associated with uterine fibroids.

\section{Supplementary materials}

Supplementary Fig. 1 can be found via https://doi.org/10.12701/ yujm.2021.01375.

\section{Notes}

\section{Conflicts of interest}

No potential conflict of interest relevant to this article was reported.

\section{Funding}

None.

\section{Author contributions}

Conceptualization: HJL. Data curation, Formal analysis: JL, HJL. Supervision: HJL. Writing-original draft: JL, HJL. Writing-review \& editing: JL, HJL.

\section{ORCID}

Jisun Lee, https://orcid.org/0000-0002-2870-3176

Hyun Jung Lee, https://orcid.org/0000-0002-3942-405X

\section{References}

1.Zimmermann A, Bernuit D, Gerlinger C, Schaefers M, Geppert K. Prevalence, symptoms and management of uterine fibroids: an international internet-based survey of 21,746 women. BMC
Womens Health 2012;12:6.

2. Hinchey J, Chaves C, Appignani B, Breen J, Pao L, Wang A, et al. A reversible posterior leukoencephalopathy syndrome. $\mathrm{N}$ Engl J Med 1996;334:494-500.

3. Casey SO, Sampaio RC, Michel E, Truwit CL. Posterior reversible encephalopathy syndrome: utility of fluid-attenuated inversion recovery MR imaging in the detection of cortical and subcortical lesions. AJNR Am J Neuroradiol 2000;21:1199-206.

4. McKinney AM, Short J, Truwit CL, McKinney ZJ, Kozak OS, SantaCruz KS, et al. Posterior reversible encephalopathy syndrome: incidence of atypical regions of involvement and imaging findings. AJR Am J Roentgenol 2007;189:904-12.

5. Magnano MD, Bush TM, Herrera I, Altman RD. Reversible posterior leukoencephalopathy in patients with systemic lupus erythematosus. Semin Arthritis Rheum 2006;35:396-402.

6. Dube M, Rathore R. Blood-transfusion-related posterior reversible encephalopathy syndrome - a description of a new case and review of the literature. Brain Circ 2020;6:269-73.

7. Nakamura Y, Sugino M, Tsukahara A, Nakazawa H, Yamamoto $\mathrm{N}$, Arawaka S. Posterior reversible encephalopathy syndrome with extensive cytotoxic edema after blood transfusion: a case report and literature review. BMC Neurol 2018;18:190.

8. Singh K, Gupta R, Kamal H, Silvestri NJ, Wolfe GI. Posterior reversible encephalopathy syndrome secondary to blood transfusion. J Clin Neurosci 2015;22:592-4.

9. Lee M, Kim TH, Kim SJ, Jee BC. Posterior reversible encephalopathy syndrome in a woman who used gonadotropin-releasing hormone agonists: a case report. Obstet Gynecol Sci 2019; 62:69-72.

10. Chakrabarti S, Morton JS, Davidge ST. Mechanisms of estrogen effects on the endothelium: an overview. Can J Cardiol 2014; 30:705-12.

11. Provenzale JM, Petrella JR, Cruz LC Jr, Wong JC, Engelter S, Barboriak DP. Quantitative assessment of diffusion abnormalities in posterior reversible encephalopathy syndrome. AJNR Am J Neuroradiol 2001;22:1455-61.

12. Bartynski WS. Posterior reversible encephalopathy syndrome, part 2: controversies surrounding pathophysiology of vasogenic edema. AJNR Am J Neuroradiol 2008;29:1043-9.

13. Bartynski WS, Boardman JF. Catheter angiography, MR angiography, and MR perfusion in posterior reversible encephalopathy syndrome. AJNR Am J Neuroradiol 2008;29:447-55.

14. Köhrmann M, Struffert T, Frenzel T, Schwab S, Doerfler A. The hyperintense acute reperfusion marker on fluid-attenuated inversion recovery magnetic resonance imaging is caused by gadolinium in the cerebrospinal fluid. Stroke 2012;43:259-61. 